\title{
El agregado de bupropion tiene la misma eficacia que el de buspirona en pacientes deprimidos que no habían respondido a citalopram
}

Adding bupropion is as effective as adding buspirone in depressed patients with no response to citalopram as first line treatment

STAR*D Study Team. N Engl J Med. 2006; 354(12):1243-52.

Objetivo

Evaluar la efectividad de la adición de bupropion o de buspirona como segundo nivel del tratamiento de la depresión.

Diseño

Ensayo clínico aleatorizado controlado.

Lugar

28 centros primarios y 23 psiquiátricos. Universidad de Texas. EE.UU.

\section{Pacientes}

565 pacientes adultos ambulatorios con trastorno depresivo mayor no psicótico, que no tuvieron remisión o no toleraron el citalopran como primera línea de tratamiento luego de 12 semanas. Fueron aleatorizados a recibir bupropion (279) ó buspirona (286) adicionalmente al citalopram y seguidos por 12 semanas.

\section{Medición de resultados principales}

La remisión sintomática fue tomada como resultado primario, ya que se asocia a un mejor funcionamiento y mejor pronóstico, definida a priori como una puntuación de cinco o menos de los 16 ítems del Inventario Rápido Autoevaluado de Sintomatología Depresiva (QIDS-C-16) que valora los síntomas centrales de la depresión mayor o una puntuación de siete o menos en la Escala de Hamilton para la Depresión (HRSD-17). La respuesta fue definida como una reducción del $50 \%$ o más de la puntuación del QIDS-C-16 a las 12 semanas con respecto a la línea de base. Las dosis usadas y su aumento escalonado pueden verse en la tabla 1.

Tabla 1: esquema del agregado de la segunda droga (mg/día) a los pacientes que no habían respondido a citalopram.

\begin{tabular}{l|c|c|c|c} 
& Dosis inicial & $\begin{array}{c}\text { Segunda } \\
\text { semana }\end{array}$ & $\begin{array}{c}\text { Tercera a quinta } \\
\text { semana }\end{array}$ & Sexta semana \\
\hline Bupropion & 200 & 200 & 300 & 400 \\
\hline Buspirona & 15 & 30 & 45 & 60 \\
\hline
\end{tabular}

Los agentes usados en segunda línea tienen diferente perfil. La buspirona es un agonista parcial post sináptico del receptor de serotonina y no es considerada como posible monoterapia. En con- traste, el bupropion bloquea la recaptación de dopamina y norepinefrina y se utiliza como monoterapia en la depresión.

\section{Resultados principales}

Las tasas y el tiempo a la remisión y respuesta fueron similares en ambos grupos y con ambas escalaslas del QIDS-C-16. Ver tabla 2.

Tabla 2: tasas de remisión y respuesta y tiempo a las mismas de acuerdo al tratamiento agregado.

\begin{tabular}{|c|c|c|c|c|c|c|}
\hline & \multicolumn{2}{|c|}{ Tasa de remisión } & \multirow[b]{2}{*}{$\begin{array}{l}\text { Tasa } \\
\text { respuesta }\end{array}$} & \multirow[b]{2}{*}{$\begin{array}{l}\text { Análisis } \\
\text { continuo* }\end{array}$} & \multicolumn{2}{|c|}{ Tiempo en semanas } \\
\hline & HRSD-17 & QIDS-C-16 & & & $\begin{array}{c}\text { A la } \\
\text { remisión }\end{array}$ & $\begin{array}{c}\text { A la } \\
\text { respuesta }\end{array}$ \\
\hline Bupropion & $29,7 \%$ & $39 \%$ & $31,8 \%$ & $25,3 \%$ & 5,2 & 6 \\
\hline Buspirona & $30,1 \%$ & $32 \%$ & $26,9 \%$ & $17 \%$ & 4,1 & 4,1 \\
\hline
\end{tabular}

Disminución respecto del puntaje basal.

No hubo diferencias significativas en la aparición de efectos adversos ni en el porcentaje de abandono del ensayo debido a los mismos.

\section{Conclusiones}

Aproximadamente $30 \%$ de los pacientes tuvieron remisión con citalopram a altas dosis (41,8 mg/día). En una segunda fase $30 \%$ de los pacientes que no habían respondido a la monoterapia con citalopram remitieron con la adición de bupropion o buspirona, aunque si se analiza en forma continua la disminución de los puntajes respecto del basal, la balanza se inclina levemente hacia el bupropion.

Estos hallazgos confirman que: 1) dos agentes pueden ser más efectivos que uno; 2) que la intolerancia o falta de eficacia de un inhibidor selectivo de la recaptación de serotonina no predice la ineficacia o intolerancia a otro.

Palabras claves: Depresión, fracaso tratamiento, antidepresivos. Key words: depression, treatment failure, antidepresives.

Fuente de financiamiento: Instituto de salud mental del Instituto de Salud de Estados Unidos.

\section{Comentario}

En los últimos años, todos los estudios clínicos y epidemiológicos realizados confirman que la depresión es un trastorno muy frecuente, en alarmante y en progresivo aumento. Es la cuarta causa de discapacidad y la Organización Mundial de la Salud estima que para el año 2.020 será la segunda, causando mayor discapacidad que la enfermedad isquémica o el accidente cerebrovascular'. Su prevalencia en Argentina oscila entre 6-7\% de la población general, estimándose en 1.500 .000 los argentinos que la padecen cada año ${ }^{2}$. En medicina ambulatoria representa un $10-20 \%$ de las consultas $^{3}$ y se estima que un $25 \%$ de la población general padece un trastorno depresivo al menos una vez en la vida.

El $80 \%$ de los pacientes con depresión no recibe tratamiento correcto o eficaz y el 20 a $30 \%$ responde mal a los tratamientos ${ }^{4}$. $\mathrm{Si}$ tenemos en cuenta estas cifras alarmantes, todo ensayo que apunte a que el tratamiento de la depresión sea más específico y por lo tanto más eficaz es no sólo atinado sino necesario.

EI STAR*D provee datos con resultados similares a los buscados en la práctica cotidiana poniendo el foco en la remisión, más que en la reducción sintomática. Sus resultados son generalizables y se pueden trasladar directamente a la práctica clínica.

Las limitaciones de éste estudio incluyen la falta de un grupo placebo control que no nos permite excluir remisiones espontáneas, o efectos no específicos de los tratamientos.

Sin embargo el STAR ${ }^{*} D$ provee numerosos datos y genera muchas preguntas. Si drogas con mecanismos de acción tan disímiles producen resultados equivalentes, como sugiere el estudio, ¿en qué apoyamos la predicción de eficacia? ¿es más importante la fisiopatologia de la depresión, contar con marcadores biológicos predictivos o la suceptibilidad individual teniendo en cuenta la genética o el medio ambiente? ¿cómo integramos ambas? La identificación de predictores específicos de respuesta a la terapia antidepresiva es aún una deuda pendiente y debería ser prioritaria para quienes financien la investigación si tenemos en cuentas su alta morbilidad y prevalencia, así como su alto costo sanitario y social. 


\section{Similar eficacia de la monoterapia con bupropion, sertralina y venlafaxina en pacientes con depresión que no habian respondido a citalopram}

Similar eficacy of bupropion, sertraline and venlafaxine monotherapy in in depressed patients with no response to citalopram as first line treatment

STAR*D Study Team. N Engl J Med. 2006;354(12):1231-42.

\section{Objetivo}

Evaluar efectividad del cambio a bupropion, sertralina o venlafaxina como segunda línea en el tratamiento de la depresión.

\section{Diseño}

Ensayo clínico, aleatorizado, controlado.

\section{Lugar}

28 centros primarios y 23 psiquiátricos. Universidad de Texas. EE.UU.

\section{Participantes}

727 adultos ambulatorios con trastorno depresivo mayor no psicótico que no tuvieron remisión o no toleraron el citalopram como primera opción de tratamiento luego de 12 semanas. Fueron aleatorizados a cambiar de antidepresivo el cual recibieron durante 14 semanas (239 bupropion, 238 sertralina, 250 venlafaxina).

\section{Medición de resultados principales}

El objetivo del tratamiento fue la remisión, definida como una puntuación de cinco o menos de los 16 ítems del Inventario Rápido Autoevaluado de Sintomatología Depresiva (QIDS-C-16) o una puntuación de siete o menos en la Escala de Hamilton para la Depresión (HRSD-17). El citalopram fue discontinuado sin período de disminución. Las dosis iniciales y subsecuentes pueden verse en la tabla 1.

Tabla 1: esquema de dosificación de cada droga en mg/día.

\begin{tabular}{l|c|c|c|c|c|c} 
& $\begin{array}{c}\text { Dosis } \\
\text { inicial }\end{array}$ & $\begin{array}{c}\mathbf{8} \text { Día } \\
\mathbf{a} \mathbf{1 4}\end{array}$ & $\begin{array}{c}\text { Dóa } \\
\mathbf{1 5} \mathbf{a} \mathbf{2 7}\end{array}$ & $\begin{array}{c}\text { Día } \\
\mathbf{2 8} \mathbf{a} \mathbf{4 1}\end{array}$ & $\begin{array}{c}\text { D́́a } \\
\mathbf{4 2} \mathbf{a} \mathbf{6 3}\end{array}$ & $\begin{array}{c}\mathbf{6 3} \text { en } \\
\text { adelante }\end{array}$ \\
\hline Bupropion & 150 & 200 & 200 & 300 & 400 & 400 \\
\hline Sertralina & 50 & 50 & 100 & 150 & 150 & 200 \\
\hline Venlafaxina & 37,5 & 75 & 150 & 225 & 300 & 375 \\
\hline
\end{tabular}

Las drogas usadas como segunda línea son diferentes entre sí. EI bupropion bloquea la recaptación de dopamina y norepinefrina; la sertralina es un inhibidor selectivo de la recaptacion de serotonina (IRSS) y la venlafaxina es un agente dual, que bloquea la recaptación de serotonina y de noradrenalina.

\section{Resultados principales}

Tanto las tasas de remisión y de respuesta, como el tiempo a las mismas no difirieron significativamente entre las distintas ramas. Ver tabla 2. La tolerabilidad y los efectos adversos fueron similares en los tres grupos.

Tabla 2: tasas de remisión y respuesta y tiempo a las mismas de acuerdo al tratamiento instituido en reemplazo de citalopram en pacientes con depresión que no habían respondido a esta droga.

\begin{tabular}{l|c|c|c|c}
\multirow{2}{*}{ Bupropion } & \multicolumn{2}{|c|}{ Tasa de remisión } & Tasa de & $\begin{array}{c}\text { Tiempo a la remisión } \\
\text { ren semanas }\end{array}$ \\
\hline respuesta & $21,3 \%$ & $25,5 \%$ & $26,1 \%$ & $5,1-4,5$ \\
\hline Sertralina & $17,6 \%$ & $26 \%$ & $26.7 \%$ & $6,2-5$ \\
\hline Venlafaxina & $24,8 \%$ & $25 \%$ & $28,2 \%$ & $5,5-4,7$ \\
\hline
\end{tabular}

\section{Conclusiones}

En pacientes sin respuesta previa a citalopram, las tasas de remisión son de aproximadamente $25 \%$, independientemente de la droga elegida en su reemplazo. La intolerancia o falta de respuesta a un IRRS no predice la ineficacia o intolerancia a otra droga de la misma familia

Palabras claves: Depresión, fracaso tratamiento, antidepresivos. Key words: depression, treatment failure, antidepresives. Fuente de financiamiento: Instituto de salud mental del Instituto de Salud de Estados Unidos.

\section{Conclusiones del comentador}

EI STAR ${ }^{\star} D$ es un estudio valioso para la práctica clínica, por la cantidad de pacientes involucrados y el tiempo total de seguimiento. Ayuda a desmitificar ciertos preceptos arraigados en la práctica y provee una conclusión desalentadora: sólo $30 \%$ de los pacientes tuvieron remisión al cabo del de la primera línea de tratamiento. Si tenemos en cuenta que el tiempo de retraso antes de recibir el primer tratamiento de depresión es en promedio seis a ocho años, las posibilidades actuales de obtener rápida remisión sintomática son aún más sombrías.

Gabriela La Monica. [ Especialista en Psiquiatría y Psicología médica. La Plata. Buenos Aires. Argentina. ]

La Monica G. Tratamiento de la Depresión: El agregado de bupropion tiene la misma eficacia que el de buspirona en pacientes deprimidos que no habían respondido a citalopram. Similar eficacia de la monoterapia con bupropion, sertralina y venlafaxina en pacientes con depresión que no habían respondido a citalopram. Evid. actual. práct. ambul; 10(1):10-11, ene-feb.2007. Comentados de: Trivedi MH, Fava M, Wisniewski SR et al; STAR ${ }^{\star}$ S Study Team. Medication augmentation after the failure of SSRIs for depression. N Engl J Med. 2006;354(12):1243-52. PMID: 16554526; y Rush AJ, Trivedi MH, Wisniewski SR, et al; STAR*D Study Team. Bupropion-SR, sertraline, or venlafaxine-XR after failure of SSRIs for depression. N Engl J Med. 2006;354(12):123142. PMID: 16554525

\section{Referencias}

1. Murray CJL, Lopez AD: Global Health Statistics: a compendium of incidence, prevalence and mortality estimates for over 200 conditions. Cambridge MA: Harvard University Press on behalf of the World Health Organization and the World Bank; 1996.

2. Rojtenberg S. (2001). Depresiones y antidepresivos. Ed Medica Panamericana.

3. Simon GE; VonKorff M. Recognition, management, and outcomes of depression in primary care. Arch Fam Med 1995 Feb;4(2):99-105.

4. Depression Guideline Panel. Depression in Primary Care: Volume 1. Detection and Diagnosis. Clinical Practice Guideline, Number 5. Rockville, MD: U.S. Department of Health and Human Services; 1993. AHCPR Publication No. 93-0550. 